\title{
Cassini radio occultations of Saturn's ionosphere: Model comparisons using a constant water flux
}

\author{
Luke Moore, ${ }^{1}$ Andrew F. Nagy, ${ }^{2}$ Arvydas J. Kliore, ${ }^{3}$ Ingo Müller-Wodarg, ${ }^{1,4}$ \\ John D. Richardson, ${ }^{5}$ Michael Mendillo ${ }^{1}$ \\ Received 16 July 2006; revised 15 September 2006; accepted 2 October 2006; published 22 November 2006.
}

[1] Recent radio occultations of Saturn's equatorial ionosphere by the Cassini spacecraft provide important insight into this poorly constrained region. Twelve new electron density profiles identify a clear dawn/dusk asymmetry as well as two apparently separate electron density peaks. This study uses a $3 \mathrm{D}$ general circulation model along with 1D water diffusion calculations to examine the possibility that a topside flux of neutral water into Saturn's atmosphere may provide a loss mechanism-via charge exchange with protons - that is sufficient to reproduce the ionosphere observed by Cassini. Results indicate that a constant influx of water of $(0.5-1.0) \times 10^{7} \mathrm{H}_{2} \mathrm{O} \mathrm{cm} \mathrm{cm}^{-2} \mathrm{sec}^{-1}$ is adequate for reproducing Cassini measurements, providing a good match to the main electron density peak at dawn and dusk. In addition, these calculations use a reduced rate for the reaction $\mathrm{H}^{+}+\mathrm{H}_{2}(\nu \geq 4) \rightarrow \mathrm{H}_{2}^{+}+\mathrm{H}$, significantly diminishing its importance in Saturn's ionospheric photochemistry. Citation: Moore, L., A. F. Nagy, A. J. Kliore, I. Müller-Wodarg, J. D. Richardson, and M. Mendillo (2006), Cassini radio occultations of Saturn's ionosphere: Model comparisons using a constant water flux, Geophys. Res. Lett., 33, L22202, doi:10.1029/2006GL027375.

\section{Introduction}

[2] The first radio occultations of Saturn's ionosphere in nearly 25 years were recently carried out by the Cassini spacecraft [Nagy et al., 2006], tripling the amount of such data. Previous measurements by Pioneer 11 in 1979 and by the Voyager spacecraft in 1980-1981 revealed a highly variable ionosphere; peak electron densities $\left(\mathrm{N}_{\mathrm{MAX}}\right)$ at dawn and dusk spanned $6000-20,000 \mathrm{~cm}^{-3}$ and the altitudes of the peak densities $\left(\mathrm{h}_{\mathrm{MAX}}\right)$ ranged from 1900 to $2900 \mathrm{~km}$ [see Atreya et al., 1984]. Twelve Cassini measurements reinforce this variability and, in addition, provide enough data to identify a clear dawn/dusk asymmetry in Saturn's equatorial ionosphere. On average, peak electron densities are larger at dusk than dawn $\left(5400 \mathrm{~cm}^{-3}\right.$

\footnotetext{
${ }^{1}$ Center for Space Physics, Boston University, Boston, Massachusetts, USA.

${ }^{2}$ Department of Atmospheric, Oceanic, and Space Sciences, University of Michigan, Ann Arbor, Michigan, USA.

${ }^{3}$ Jet Propulsion Laboratory, California Institute of Technology, Pasadena, California, USA.

${ }^{4}$ Department of Physics and Astronomy, Imperial College, London, UK.

${ }^{5}$ Kavli Institute for Astrophysics and Space Research, Massachusetts Institute of Technology, Cambridge, Massachusetts, USA.
}

Copyright 2006 by the American Geophysical Union. 0094-8276/06/2006GL027375 vs. $1700 \mathrm{~cm}^{-3}$ ) and the peak altitudes are lower at dusk than dawn $(1880 \mathrm{~km}$ vs. $2360 \mathrm{~km})$ [Nagy et al., 2006].

[3] Early photochemical models of the outer planets' atmospheres predicted ionospheres dominated by $\mathrm{H}^{+}$, as there was no known mechanism to effectively convert longlived protons to short-lived molecular ions in a hydrogen atmosphere [e.g., McElroy, 1973]. Electron density predictions from such model calculations proved to be too large by an order of magnitude; however, McElroy did point out that the charge-exchange reaction of $\mathrm{H}^{+}$with $\mathrm{H}_{2}$ would become exothermic for vibrational levels 4 and higher. For Jupiter, McConnell et al. [1982] were able to reproduce most of the ionospheric profiles measured by the Pioneer and Voyager spacecraft by utilizing an $\mathrm{H}_{2}$ vibrational distribution enhanced significantly above LTE and by forcing vertical motion of the plasma. Similar matches were subsequently shown to be possible at Saturn as well [Majeed and McConnell, 1991; Moses and Bass, 2000]. These Saturn studies also evaluated the effects of an influx of $\mathrm{H}_{2} \mathrm{O}$ into the atmosphere, which can charge-exchange with $\mathrm{H}^{+}$creating short-lived molecular ions, thus reducing the electron density, as first proposed by Connerney and Waite [1984].

[4] Determination of the exact vibrational distribution of molecular hydrogen in Saturn's thermosphere is difficult, however. A thorough overview of this problem is given by Yelle and Miller [2004]. Recently, Huestis [2005] pointed out that previous calculations of $\mathrm{H}_{2}$ vibrational distributions did not include an important relaxation reaction, $\mathrm{H}^{+}+$ $\mathrm{H}_{2}\left(\nu_{\mathrm{i}}\right) \rightarrow \mathrm{H}^{+}+\mathrm{H}_{2}\left(\nu_{\mathrm{f}}<\nu_{\mathrm{i}}\right)$, which could reduce the calculated population of all the vibrationally excited levels by at least a factor of 2 [e.g., Krstić, 2002].

[5] Identifying the source of the water that, ultimately, ends up in Saturn's atmosphere is similarly difficult. Saturn's rings are predominantly water ice, and both neutral [Esposito et al., 2005] and plasma [Young et al., 2005] water products have been observed near the rings by Cassini. Monte Carlo modeling results constrained primarily by Hubble Space Telescope (HST) measurements of Saturn's $\mathrm{OH}$ torus demonstrated that a strong source of $\mathrm{H}_{2} \mathrm{O}$ was concentrated near Enceladus in the tenuous E-ring [Jurac and Richardson, 2005]. Cassini's recent discovery of a gaseous plume near Enceladus' southern pole [e.g., Porco et al., 2006; Waite et al., 2006] reinforces the notion that the bulk of water in the Saturn system could be coming from the region surrounding Enceladus, and thus may enter Saturn's atmosphere as a neutral. Alternatively, Prangé et al. [2006] use HST observations to argue that the presence of stratospheric water is consistent with localized influxes of water, which result from 


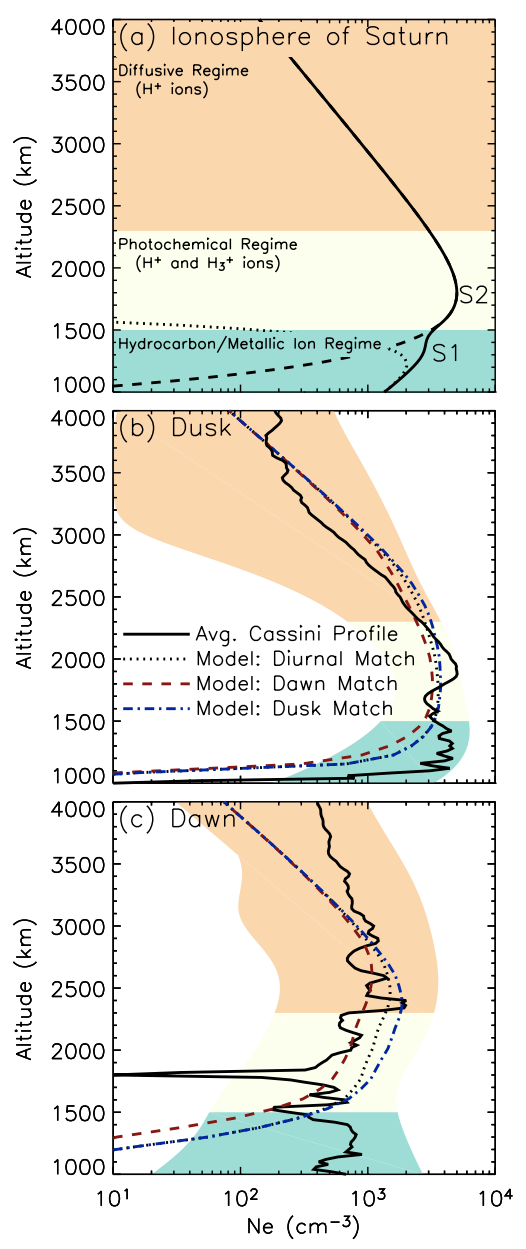

Figure 1. (a) A schematic of Saturn's ionosphere, broken into three distinct regimes. The double-peak structure, identified here as S1 and S2, can be seen in recent Cassini radio occultations, and likely results from different ion compositions. Plots of the average (b) dusk and (c) dawn Cassini electron density profiles along with the best fit model calculations are shown (see Sections 3 and 4). The dotted lines represent model results that best match the observations at both dusk and dawn using a full diurnal calculation with a single set of parameters: $k=0.25 k_{1}$, and $\Phi_{\mathrm{H}_{2} \mathrm{O}}=5 \times 10^{6}$ molecules $\mathrm{cm}^{-2} \mathrm{sec}^{-1}$. Dashed lines give model results best matched to the average dawn profile: $k=$ $0.5 k_{1}$, and $\Phi_{\mathrm{H}_{2} \mathrm{O}}=1 \times 10^{7}$ molecules $\mathrm{cm}^{-2} \mathrm{sec}^{-1}$. Dotdashed lines give model results best matched to the average dusk profile: $k=0.15 k_{1}$, and $\Phi_{\mathrm{H}_{2} \mathrm{O}}=5 \times 10^{6}$ molecules $\mathrm{cm}^{-2} \mathrm{sec}^{-1}$. The width of the shaded regions corresponds to the full range of electron densities observed by Cassini, and the degrees of shading correspond to the ionospheric regimes identified in Figure 1a.

ionized ring particles traveling along magnetic field lines into Saturn's atmosphere [Connerney, 1986].

\section{Approach to the Problem}

[6] In order to understand Saturn's highly variable ionosphere, it is important to highlight the observed consistencies and build upon them. First, there is a clear dawn/dusk asymmetry in peak electron density and altitude. This asymmetry is addressed here by converting atomic ions to molecular ions, the mechanisms and consequences of which are described in Section 4. Second, Cassini measurements of Saturn's equatorial ionosphere [Nagy et al., 2006, Figures 3 and 4] reveal a possible double peak structure in electron density. This structure is illustrated schematically in Figure 1a, specifying the two peaks as S1 and S2, and separating Saturn's ionosphere into three regimes each governed by different photochemistry and plasma diffusion.

[7] The densities and altitudes of Figure 1a are chosen to represent a "typical" ionospheric profile, although there is certainly considerably variability that cannot be captured within a single schematic. The secondary ion peak (S1) is at too low an altitude to be dominated by Saturn's most numerous ions in photochemical equilibrium (i.e., $\mathrm{H}^{+}$and $\mathrm{H}_{3}^{+}$); based on the magnitude and altitude of $\mathrm{S} 1$, it seems likely to be comprised mostly of hydrocarbon and/or metallic ions [Moses and Bass, 2000]. It is also possible that gravity wave interactions can lead to such a dramatic layering in the electron density profile [Matcheva and Strobel, 2001]. The region above S1, where electron densities are generally larger, compares well with previous estimates for an ionosphere dominated by $\mathrm{H}^{+}$and $\mathrm{H}_{3}^{+}$[e.g., Majeed and McConnell, 1996]. Diffusive processes eventually begin to take precedence over photochemistry, however, and plasma transport analysis is required at higher altitudes [Moore et al., 2004].

\section{Observations and Modeling}

[8] A detailed description of radio occultations of the Saturn ionosphere made by Cassini's Radio Science Subsystem (RSS) instrument appears in the work of Nagy et al. [2006]. Between 2 May and 5 September 2005, twelve ionospheric radio occultations were recorded: seven dawn measurements and five dusk measurements. Latitudes ranged between $9^{\circ} \mathrm{S}$ and $7.1^{\circ} \mathrm{N}$, at solar zenith angles between $84^{\circ}$ and $96^{\circ}$. Average dawn and dusk profiles are given in Figures $1 \mathrm{~b}$ and $1 \mathrm{c}$, as well as several model fits to those profiles, as described in Section 4.

[9] The dawn and dusk profiles given in Figures $1 \mathrm{~b}$ and $1 \mathrm{c}$ represent averaged behaviors, with the horizontal extent of the shading giving the full range of electron densities observed at each altitude [Nagy et al., 2006]. While it is clear that the averaged profiles of Figures $1 \mathrm{~b}$ and $1 \mathrm{c}$ cannot fully represent the wide variations in altitude and density observed by Cassini, they do display two properties common to all of the individual ingress-egress occultation pairs: dawn profiles have smaller peak densities and higher peak altitudes than dusk profiles. In addition, these properties are largely independent of the method by which the "average" is constructed. For example, the profiles in Figures $1 \mathrm{~b}$ and $1 \mathrm{c}$ use a weighted sum of electron density profiles measured at dawn and dusk by Cassini; they result in dawn/dusk $\mathrm{N}_{\mathrm{MAX}}$ values of $1700 / 5400 \mathrm{e}^{-} \mathrm{cm}^{-3}$ and $\mathrm{h}_{\text {MAX }}$ values of $2360 / 1880 \mathrm{~km}$. If, instead, the mean of the upper peak is calculated from Nagy et al. [2006, Table 1], then the dusk/dawn $\mathrm{N}_{\mathrm{MAX}}$ variation is 1480 / $4090 \mathrm{e}^{-} \mathrm{cm}^{-3}$ and the $\mathrm{h}_{\text {MAX }}$ variation is $2580 / 1960 \mathrm{~km}$. 


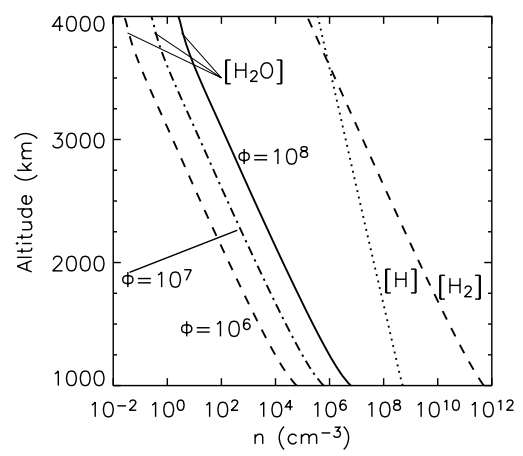

Figure 2. Thermospheric densities from STIM used in this study. In addition, water density profiles from 1D neutral diffusion calculations are given, resulting from the topside water influxes indicated on the plot (in units of $\mathrm{H}_{2} \mathrm{O}$ molecules $\mathrm{cm}^{-2} \mathrm{sec}^{-1}$ ).

[10] The Saturn-Thermosphere-Ionosphere-Model (STIM) is a general circulation model (GCM) of Saturn's upper atmosphere. Initial thermospheric results are described by Müller-Wodarg et al. [2006] and ionospheric results by Moore et al. [2004]. Solar irradiances in those studies were specified using the SOLAR2000 empirical model [Tobiska and Bouwer, 2006] under representative solar maximum and minimum conditions. This study employs the most recent STIM thermosphere, which reproduces the observed exospheric temperatures at Saturn using a combination of joule heating in the auroral zones and a wave heating profile peaked at the equator to supply the additional energy not provided by solar heating alone. Specifically, the thermosphere from Simulation 7 of Müller-Wodarg et al. [2006, Table 1] is used, except for solstice rather than equinox conditions.

[11] In order to evaluate the effects of a downward flux of water on Saturn's ionosphere, 1D neutral diffusion calculations are performed with the STIM thermosphere using a constant flux at the topside as the boundary condition. The altitude-dependent water flux is given by

$$
\phi=-D\left(\frac{\partial n}{\partial z}+\frac{n}{T_{n}} \frac{\partial T_{n}}{\partial z}+\frac{n}{H}\right)
$$

where $D$ is the neutral diffusion coefficient of $\mathrm{H}_{2} \mathrm{O}$ through $\mathrm{H}_{2}, n$ the water density, $T_{n}$ the neutral temperature, and $H$ the water scale height. The other constituents in the STIM thermosphere are held fixed while $\mathrm{H}_{2} \mathrm{O}$ diffuses according to a numerical solution of its continuity equation. The neutral atmosphere used in this study, along with three sample water density profiles, is given in Figure 2 in order to convey some quantitative sense of the background parameters to the reader. For example, a water mixing ratio of $\sim 6 \times 10^{-7}$ results from a topside flux of $1 \times 10^{7} \mathrm{H}_{2} \mathrm{O}$ $\mathrm{cm}^{-2} \mathrm{sec}^{-1}$. The constant downward flux of water leads to a water density profile that is proportional to the density profile of Saturn's atmosphere. This time-dependent numerical result is consistent with analytical derivations of a steady-state mixing distribution for a minor constituent in an isothermal atmosphere [e.g., Connerney and Waite, 1984; Bauer, 1973].
[12] Ionospheric calculations are performed using the photochemistry and plasma diffusion methods described by Moore et al. [2004]. The Cassini $\mathrm{Ne}(\mathrm{h})$ profiles obtained to-date are all near equatorial, and these present a special case for modeling plasma diffusion. Vertical motions in this region are not diffusive, but electro-dynamic, while horizontal diffusion is meridional along the field lines that result from a magnetic dipole co-aligned with Saturn's rotational axis. Below a particular altitude, however, Saturn's ionosphere is in photochemical equilibrium, and dynamics may be ignored [Moore et al., 2004]. The altitude of this boundary will vary; $2300 \mathrm{~km}$ is typical for the conditions sampled by Cassini. Assuming the lower ionospheric peak (S1) results from metallic and/or hydrocarbon ions, with possible contributions resulting from upward propagating gravity waves - processes not included in this model calculations in this study seek only to compare directly with Cassini observations within the hydrogen photochemical regime, where the main ionospheric peak (S2) occurs.

[13] In an attempt to fully explore the available parameter space in Saturn's ionosphere, the calculations investigate a wide range of solar irradiances, water fluxes, latitudes, and rates for the reaction $\mathrm{H}^{+}+\mathrm{H}_{2}(\nu \geq 4) \rightarrow \mathrm{H}_{2}^{+}+\mathrm{H}$ (called reaction $k_{1}$ by Moore et al. [2004]). Three alternatives of solar irradiance averaged over the eight days of Cassini occultations are explored: SOLAR2000 v1.23, v2.27, and the EUVAC model [Richards et al., 1994]. It is worth noting that, despite variations by up to an order of magnitude at specific wavelengths, the overall solar irradiances given by SOLAR2000 v2.27 and EUVAC yield nearly identical model ionospheres. A wide range of water fluxes, spanning $10^{5}-10^{8} \mathrm{H}_{2} \mathrm{O} \mathrm{cm} \mathrm{cm}^{-2} \mathrm{sec}^{-1}$, is evaluated. Twelve values of the $k_{1}$ rate are investigated, bound by a null $k_{1}$ rate at one end and a value 100 times the nominal rate at the other end. The nominal $k_{1}$ rate is identical to that described by Moore et al. [2004], i.e., $7.5 \times 10^{-14} \mathrm{~cm}^{-3} \mathrm{sec}^{-1}$ above $2000 \mathrm{~km}$, and ranging from $(1$ to 750$) \times 10^{-16} \mathrm{~cm}^{-3} \mathrm{sec}^{-1}$ below. Calculations are made for three different latitudes: the average latitude for Cassini's seven dawn occultations $\left(-5.5^{\circ}\right)$, the average for five dusk occultations $\left(-1.5^{\circ}\right)$, and the global average $\left(-3.5^{\circ}\right)$. In all, over 1000 separate runs were performed using different combinations of the above parameters.

\section{Results and Discussion}

[14] In the absence of proton loss via charge exchange with $\mathrm{H}_{2}$ and/or $\mathrm{H}_{2} \mathrm{O}$, the photochemically produced peak in Saturn's ionosphere is dominated by $\mathrm{H}^{+}$and located between $\sim 1000-1500 \mathrm{~km}$ with very little diurnal variation [e.g., Moore et al., 2004]. At higher altitudes, the dominance of $\mathrm{H}^{+}$over other ion species in Saturn's ionosphere - and hence, the lack of diurnal variation - is even more dramatic. By accounting for loss mechanisms that convert protons into short-lived molecular ions, the electron density peak is reduced in magnitude and occurs at a higher altitude. The reduction in $\mathrm{N}_{\mathrm{MAX}}$ is due directly to the fact that protons charge-exchange with $\mathrm{H}_{2}$ or $\mathrm{H}_{2} \mathrm{O}$, creating a molecular ion that recombines quickly, thereby reducing the net ion/electron density. The increase in $h_{\operatorname{MAX}}$ occurs because the rate of charge-exchange for $\mathrm{H}^{+}$is largest near the photochemical electron peak, as there are more ions and 
Table 1. Best Model Fits to Cassini Data for Variable $k_{1}$ Rate $^{\mathrm{a}}$

\begin{tabular}{|c|c|c|c|c|c|c|}
\hline \multirow[b]{2}{*}{$k$ Rate $\left(\times k_{1}\right)^{\mathrm{b}}$} & \multicolumn{2}{|c|}{ Dawn } & \multicolumn{2}{|c|}{ Dusk } & \multicolumn{2}{|c|}{ Diurnal } \\
\hline & $\Phi_{\mathrm{H}_{2} \mathrm{O}}, \mathrm{cm}^{-2} \mathrm{~s}^{-1}$ & $\frac{\sum\left(N_{\text {model }}-N_{\text {data }}\right)^{2 \mathrm{c}}}{M I N_{\text {dawn }}}$ & $\Phi_{\mathrm{H}_{2} \mathrm{O}}, \mathrm{cm}^{-2} \mathrm{~s}^{-1}$ & $\frac{\sum\left(N_{\text {model }}-N_{\text {data }}\right)^{2 \mathrm{c}}}{M I N_{\text {dusk }}}$ & $\Phi_{\mathrm{H}_{2} \mathrm{O}}, \mathrm{cm}^{-2} \mathrm{~s}^{-1}$ & $\frac{\Sigma\left(N_{\text {model }}-N_{\text {data }}\right)^{2 \mathrm{c}}}{M_{\text {diurnal }}}$ \\
\hline 0 & $5 \times 10^{7}$ & 2.96 & $1 \times 10^{7}$ & 4.17 & $2.5 \times 10^{7}$ & 2.77 \\
\hline 0.05 & $5 \times 10^{7}$ & 3.12 & $1 \times 10^{7}$ & 2.52 & $1 \times 10^{7}$ & 2.38 \\
\hline 0.15 & $2.5 \times 10^{7}$ & 1.82 & $5 \times 10^{6}$ & 1.00 & $1 \times 10^{7}$ & 1.26 \\
\hline 0.25 & $2.5 \times 10^{7}$ & 1.67 & $5 \times 10^{6}$ & 2.42 & $5 \times 10^{6}$ & 1.00 \\
\hline 0.5 & $1 \times 10^{7}$ & 1.00 & $1 \times 10^{6}$ & 4.76 & $5 \times 10^{6}$ & 1.26 \\
\hline 0.75 & $5 \times 10^{6}$ & 1.14 & $1 \times 10^{6}$ & 5.86 & $5 \times 10^{6}$ & 1.57 \\
\hline 1 & $5 \times 10^{6}$ & 1.19 & $1 \times 10^{6}$ & 6.68 & $1 \times 10^{6}$ & 1.70 \\
\hline 1.5 & $5 \times 10^{6}$ & 1.44 & $1 \times 10^{5}$ & 7.74 & $1 \times 10^{6}$ & 1.91 \\
\hline 2 & $5 \times 10^{6}$ & 1.63 & $1 \times 10^{5}$ & 8.50 & $1 \times 10^{5}$ & 2.06 \\
\hline 5 & $5 \times 10^{6}$ & 2.02 & $1 \times 10^{5}$ & 10.6 & $1 \times 10^{5}$ & 2.53 \\
\hline 10 & $1 \times 10^{6}$ & 2.07 & $1 \times 10^{5}$ & 11.5 & $1 \times 10^{5}$ & 2.74 \\
\hline 100 & $1 \times 10^{6}$ & 1.66 & $1 \times 10^{5}$ & 12.2 & $1 \times 10^{5}$ & 2.88 \\
\hline
\end{tabular}

a"Best" model fits are found by varying the water flux in order to minimize the model's deviation from the data for a given value of $k$. Bold identifies the modeled "best fit" for dawn, dusk, and for both dawn and dusk simultaneously. Electron density profiles from the bold fits are given in Figures $1 \mathrm{~b}$, 1c, and 3 .

${ }^{\mathrm{b}}$ The nominal $k_{1}$ rate is identical to that used by Moore et al. [2004]: $7.5 \times 10^{-14} \mathrm{~cm}^{-3} \mathrm{sec}^{-1}$ above $2000 \mathrm{~km}$, and spanning $(1 \mathrm{to} 750) \times 10^{-16} \mathrm{~cm}^{-3} \mathrm{sec}{ }^{-1}$ between 1000 and $2000 \mathrm{~km}$.

${ }^{\mathrm{c}}$ The sum of the squares of the differences, normalized by the respective minimum value. The absolute magnitude of this value is unimportant; only its relative variation has meaning.

more neutral molecules at those altitudes. Thus, the rate of electron loss is larger at lower altitudes, and the net effect is to shift $\mathrm{h}_{\mathrm{MAX}}$ to higher altitudes. In this way, a water flux can affect both the altitude and density of the ionospheric peak.

[15] Table 1 summarizes some of the model calculations by describing the water flux necessary to reproduce Cassini observations for the full range of $k_{1}$ reaction rates. Values in Table 1 are for EUVAC solar fluxes at $-3.5^{\circ}$ latitude. Each row represents a series of calculations conducted using the specified value for $k_{1}$. The dawn, dusk, and diurnal water fluxes identified indicate the values that provided the best match to the observations, based on the selected $k_{1}$ rate. The quality of each match is determined by summing the squares of the differences between the modeled and observed profile within the photochemical regime (light shading, Figure 1a). Figures $1 \mathrm{~b}$ and $1 \mathrm{c}$ give the average dawn and dusk electron density profiles observed by Cassini from May-September 2005 , along with the best fit model profiles from this study. The best model fit parameters for $k$ and $\Phi_{\mathrm{H}_{2} \mathrm{O}}$ for a dawn, dusk, and diurnal match are identified by bold font in Table 1; these are the respective profiles drawn in Figures $1 \mathrm{~b}$ and $1 \mathrm{c}$.

[16] The observed dawn/dusk asymmetry can be modeled using a constant water flux. Primarily this asymmetry is due to the presence of both an atomic $\left(\mathrm{H}^{+}\right)$and a molecular $\left(\mathrm{H}_{3}^{+}\right)$ion at the electron density peak. At dusk the solar source of ionization has only just shut off, so both $\mathrm{H}^{+}$and $\mathrm{H}_{3}^{+}$ions are still present to contribute to the electron density peak. However, during the five hours of darkness on the nightside, most of the $\mathrm{H}_{3}^{+}$ions have recombined, resulting in a reduced electron density at dawn. This effect is similar to that observed in another well-known atomic and molecular ion region-the terrestrial F-layer. Without including an additional loss process due to charge-exchange with $\mathrm{H}_{2}$ and/or $\mathrm{H}_{2} \mathrm{O}$, the $\mathrm{H}_{3}^{+}$ion mixing ratio at the electron density peak would be too low to cause the observed dawn/dusk asymmetry.
[17] Modeled diurnal variations of $\mathrm{N}_{\mathrm{MAX}}$ resulting from the parameters identified in the "Diurnal" column of Table 1 are plotted in Figure 3. The thick solid line gives the local time variation of the best fit electron density peak in this study $\left(k=0.25 k_{1}, \Phi_{\mathrm{H} 2 \mathrm{O}}=5 \times 10^{6} \mathrm{~cm}^{-2} \mathrm{sec}^{-1}\right)$. It can be seen from Figure 3 that a single set of parameters can produce an ionosphere that displays the asymmetry present in the Cassini dawn/dusk averages, which are identified by an " $x$ " on the plot.

[18] Model calculations are best able to reproduce Cassini observations when a steady-state water flux between $(0.5-$ 1.0) $\times 10^{7} \mathrm{~cm}^{-2} \mathrm{sec}^{-1}$ is assumed. These values fall within the boundaries of previous estimates, and differ sensibly from them based on the different assumptions used. For example, the larger planet-wide influx of $4 \times 10^{7} \mathrm{H}_{2} \mathrm{O}$

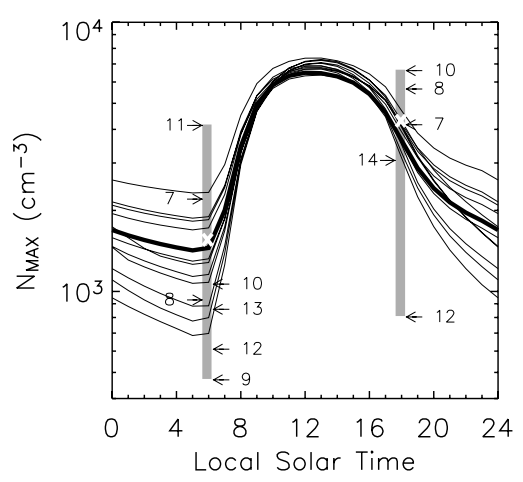

Figure 3. Plot of calculated local time variations of $\mathrm{N}_{\mathrm{MAX}}$. Each solid line represents the best diurnal match for one row in Table 1 ( $k$ and $\Phi \mathrm{H}_{2} \mathrm{O}$ ). The best fit (bold valves, "Diurnal" column) given as the thick solid line. Grey shaded regions identify the ranges in LT and $\mathrm{N}_{\text {MAX }}$ from the upper (S2) peak of Cassini's twelve occultations. Numbers mark the individual occultation values and " $\mathrm{x}$ " marks the averaged dawn and dusk observation [Nagy et al., 2006, Table 1]. 
$\mathrm{cm}^{-2} \mathrm{sec}^{-1}$ quoted by Connerney and Waite [1984] is explained by the absence of reaction $k_{1}$ in their calculations. Similarly, a water flux of $\sim 6 \times 10^{6} \mathrm{H}_{2} \mathrm{O} \mathrm{cm} \mathrm{cm}^{-2} \mathrm{sec}^{-1}$ is expected from recent Monte Carlo simulations that predict a source of $\sim 10^{28} \mathrm{H}_{2} \mathrm{O} \mathrm{sec}^{-1}$ centered near the orbit of Enceladus [Jurac and Richardson, 2005]; subsequent calculations estimate that $14 \%$ of the $\mathrm{H}_{2} \mathrm{O}$ produced at Enceladus is lost to Saturn, and most of that in the equatorial plane. Finally, the $1.5 \times 10^{6} \mathrm{H}_{2} \mathrm{O} \mathrm{cm} \mathrm{cm}^{-2} \mathrm{sec}^{-1}$ water flux determined by Moses et al. [2000] was derived from infrared observations at $30^{\circ} \mathrm{N}$ latitude [cf. Feuchtgruber et al., 1997], whereas larger water densities are expected at the equatorial latitudes sampled by Cassini [Jurac and Richardson, 2005]. Still, what is unclear at present is how realistic a time-independent water flux is. If most of the water is coming from the plume on Enceladus' southern pole [e.g., Porco et al., 2006], or is sputtered from Saturn's rings, then it seems likely to be a time-variable flux, a fact that more observations by Cassini should help resolve.

[19] In a study of 3D neutral diffusion in Earth's atmosphere, Bernhardt [1979] derived an analytical approximation for the gas concentration resulting from a pointsource release of neutral gas. Calculations using this approximation in Saturn's atmosphere, and taking the point of "release" to be the upper atmosphere (e.g., $4000 \mathrm{~km}$, representing a downward water flux), show that it takes $\sim 10$ hours for water to diffuse through Saturn's thermosphere to the photochemical peak, $\sim 1500 \mathrm{~km}$. Timedependent neutral water diffusion calculations from this study reproduce this result, meaning that the $\mathrm{H}_{2} \mathrm{O}$ density profile is steady for essentially the entire ionosphere after $\sim 10$ hours. Thus, if the water influx at the top of Saturn's atmosphere is constant on a 10-hour timescale, it is a good approximation to use a steady-state water flux. Calculations that involve time-dependent water fluxes are the subject of future study.

[20] Other than from radio occultations, the only ionospheric measurements available at Saturn are from planetary lightning, called Saturn Electric Discharge (SED). By noting the cutoff frequency of Voyager 2 SED observations, Kaiser et al. [1984] inferred a diurnal noon-to-midnight variation in $\mathrm{N}_{\mathrm{MAX}}$ of 2 orders of magnitude, from $10^{5} \mathrm{~cm}^{-3}$ to $10^{3} \mathrm{~cm}^{-3}$. Such a variation is far in excess of any produced by ionospheric models-including that of Figure 3, which is roughly a factor of 3 [e.g., Majeed and McConnell, 1996; Moses and Bass, 2000; Moore et al., 2004]. Ring shadowing effects could also contribute to resolving this discrepancy by creating radio frequency "windows" in Saturn's ionosphere [Mendillo et al., 2005]. Cassini may shed new light on the topic by providing more SED data, and by providing enough dawn/dusk radio occultation profiles to constrain ionospheric models at those local times. Future validation of the Saturn-ThermosphereIonosphere-Model (STIM) depends critically on additional Cassini observations at various latitudes and local times from radio occultations and related experiments.

[21] Acknowledgments. STIM development at Boston University was funded by the NASA Planetary Atmospheres program, which also supported JDR. LM acknowledges support from a NASA Graduate Student Researchers Program fellowship, and IM-W is supported by a British Royal Society Fellowship. AJK and AFN acknowledge support from the Cassini Project Office.

\section{References}

Atreya, S. K., J. H. Waite, T. M. Donahue, A. F. Nagy, and J. C. McConnell (1984), Theory, measurements, and models of the upper atmosphere and ionosphere of Saturn, in Saturn, edited by T. Gehrels and M. S. Matthews, pp. 239-277, Univ. of Ariz. Press, Tucson.

Bauer, S. J. (1973), Physics of Planetary Ionospheres, Springer, New York. Bernhardt, P. A. (1979), Three-dimensional, time-dependent modeling of neutral gas diffusion in a nonuniform, chemically reactive atmosphere, J. Geophys. Res., 84, 793-802.

Connerney, J. E. P. (1986), Magnetic connection for Saturn's rings and atmosphere, Geophys. Res. Lett., 13, 773-776.

Connerney, J. E. P., and J. H. Waite Jr. (1984), New model of Saturn's ionosphere with an influx of water from the rings, Nature, 312, 136-138.

Esposito, L. W., et al. (2005), Ultraviolet imaging spectroscopy shows an active Saturnian system, Science, 307, 1251-1255.

Feuchtgruber, H., E. Lellouch, T. de Graauw, B. Bézard, T. Encrenaz, and M. Griffin (1997), External supply of oxygen to the atmospheres of the giant planets, Nature, 389, 159-162.

Huestis, D. L. (2005), $\mathrm{H}^{+}+\mathrm{H}_{2}$ ion-molecule reactions in the ionospheres of the outer planets, Bull. Am. Astron. Soc., 37, 757.

Jurac, S., and J. D. Richardson (2005), A self-consistent model of plasma and neutrals at Saturn: Neutral cloud morphology, J. Geophys. Res., 110, A09220, doi:10.1029/2004JA010635

Kaiser, M. L., M. D. Desch, and J. E. P. Connerney (1984), Saturn's ionosphere: Inferred electron densities, J. Geophys. Res., 89, 2371-2376.

Krstić, P. S. (2002), Inelastic processes from vibrationally excited states in slow $\mathrm{H}^{+}+\mathrm{H}_{2}$ and $\mathrm{H}+\mathrm{H}_{2}$ collisions: Excitations and charge transfer, Phys. Rev. A, 66, 042717, doi:10.1103/PhysRevA.66.042717.

Majeed, T., and J. C. McConnell (1996), Voyager electron density measurements on Saturn: Analysis with a time dependent ionospheric model, J. Geophys. Res., 101, 7589-7598.

Majeed, T., and J. C. McConnell (1991), The upper ionospheres of Jupiter and Saturn, Planet. Space Sci., 39, 1715-1732.

Matcheva, K. I., and D. F. Strobel (2001), Interaction of gravity waves with ionospheric plasma: Implications for Jupiter's ionosphere, Icarus, 152, $347-365$

McConnell, J. C., J. B. Holberg, G. R. Smith, B. R. Sandel, D. E. Shemansky, and A. L. Broadfoot (1982), A new look at the ionosphere of Jupiter in light of the UVS occultation results, Planet. Space Sci., 30, 151-167.

McElroy, M. B. (1973), Ionospheres of the major planets, Space Sci. Rev., 14, 460-473.

Mendillo, M., L. Moore, J. Clarke, I. Mueller-Wodarg, W. S. Kurth, and M. L. Kaiser (2005), Effects of ring shadowing on the detection of electrostatic discharges at Saturn, Geophys. Res. Lett., 32, L05107, doi:10.1029/2004GL021934.

Moore, L. E., M. Mendillo, I. C. F. Müller-Wodarg, and D. L. Murr (2004), Modeling of global variations and ring shadowing in Saturn's ionosphere, Icarus, 172, 503-520.

Moses, J. I., and S. F. Bass (2000), Effects of external material on the chemistry and structure of Saturn's ionosphere, J. Geophys. Res., 105, 7013-7052

Moses, J. I., B. Bézard, E. Lellouch, G. R. Gladstone, H. Feuchtgruber, and M. Allen (2000), Photochemistry of Saturn's atmosphere, II, Effects of an influx of external oxygen, Icarus, 145, 166-202.

Müller-Wodarg, I. C. F., M. Mendillo, R. V. Yelle, and A. D. Aylward (2006), A global circulation model of Saturn's thermosphere, Icarus, $180,147-160$.

Nagy, A. F., et al. (2006), First results from the ionospheric radio occultations of Saturn by the Cassini spacecraft, J. Geophys. Res., 111, A06310, doi:10.1029/2005JA011519.

Porco, C. C., et al. (2006), Cassini observes the active South Pole of Enceladus, Science, 311, 1393-1401.

Prangé, R., T. Fouchet, R. Courtin, J. E. P. Connerney, and J. C. McConnell (2006), Latitudinal variation of Saturn photochemistry deduced from spatially-resolved ultraviolet spectra, Icarus, 180, 379-392.

Richards, P. G., J. A. Fennelly, and D. G. Torr (1994), EUVAC: A solar EUV flux model for aeronomic calculations, J. Geophys. Res., 99, 8981-8992.

Tobiska, W. K., and S. D. Bouwer (2006), New developments in SOLAR2000 for space research and operations, Adv. Space Res., 37, 347-358.

Waite, J. H., et al. (2006), Cassini Ion and Neutral Mass Spectrometer: Enceladus plume composition and structure, Science, 311, 1419-1422.

Yelle, R. V., and S. Miller (2004), Jupiter's thermosphere and ionosphere, in Jupiter: The Planet, Satellites, and Magnetosphere, edited by F. Bagenal et al., pp. 185-218, Cambridge Univ. Press, New York.

Young, D. T., et al. (2005), Composition and dynamics of plasma in Saturn's magnetosphere, Science, 307, 1262-1266.

A. J. Kliore, Jet Propulsion Laboratory, California Institute of Technology, MS 161-260, Pasadena, CA 91109, USA. 
M. Mendillo and L. Moore, Center for Space Physics, Boston University, 725 Commonwealth Ave., Boston, MA 02215, USA (moore@bu.edu)

I. Müller-Wodarg, Department of Physics and Astronomy, Imperial College, 67-73 Riding House St., London W1P 7PP, UK.
A. F. Nagy, Department of Atmospheric, Oceanic, and Space Sciences, University of Michigan, 1422 Space Research Bldg., Ann Arbor, MI 48109, USA.

J. D. Richardson, Kavli Institute for Astrophysics and Space Research, Massachusetts Institute of Technology, 77 Massachusetts Ave., Bldg. $37-$ 655, Cambridge, MA 02139, USA. 